\title{
Using Radial Basis Functions to Solve Geodesics Equations for Body Measurements
}

\author{
R. $\mathrm{Ng}^{1+}$, G.T.Y. Pong ${ }^{2}$ and M. Wong ${ }^{2}$ \\ ${ }^{1}$ Institute of Textiles and Clothing, Hong Kong Polytechnic University, Hong Kong SAR, China \\ ${ }^{2}$ Department of Applied Mathematics, Hong Kong Polytechnic University, Hong Kong SAR, China
}

( Received 4 October 2006, accepted 4 February 2007)

\begin{abstract}
For the application of apparel industry, 3D body measurements are the minimal arc length distances between the point couples along the body surface. The calculation of geodesics is nontrivial, and can only be approximated for free-form surfaces, because the computation of geodesics requires solving a set of ordinary differential equations (geodesics equations), which do not exhibit any closed form solution for free-form surfaces. In this article, the method of radial basis function (RBF) is used in solving the geodesics equations based on the bicubic tensor product Bezier patches. This method does not require any mesh to support the localized approximations. The accuracy and efficiency of this meshless collocation method are examined by comparison to the Ritz's method.
\end{abstract}

Keywords: Geodesics, Radial Basis Functions

\section{Introduction}

The introduction is divided into three parts: the background of solving the geodesics equations, the application of geodesics equation in the apparel industry, and the focus of the current research work.

1) Background of solving geodesic equations: Geodesics are defined in the differential geometry literature as the locally shortest path between two points on a surface. The trajectory of the geodesic depends on the intrinsic properties of the surface. A local geodesic satisfies a pair of ODEs, (1) and (2), defined using the Christoffel symbols, $\Gamma^{\mathrm{i}} \mathrm{jk}$. The complexity of solving the geodesic equations depends on the definition of the surface. Typically, the solutions of geodesics on free-form surfaces do not have any closed form solution. For this reason, numerical method must be used to approximate the value.

$$
\begin{aligned}
& G E_{l}(u, v)=u^{\prime \prime}+\Gamma^{1}{ }_{11}\left(u^{\prime}\right)^{2}+2 \Gamma^{1}{ }_{12} u^{\prime} v^{\prime}+\Gamma^{1}{ }_{22}\left(v^{\prime}\right)^{2}=0 \\
& G E_{2}(u, v)=v^{\prime \prime}+\Gamma^{2}{ }_{11}\left(u^{\prime}\right)^{2}+2 \Gamma^{2}{ }_{12} u^{\prime} v^{\prime}+\Gamma^{2}{ }_{22}\left(v^{\prime}\right)^{2}=0
\end{aligned}
$$

The numerical methods being used can be typically classified into two classes according to whether the surface is continuous or discrete. In solving the geodesics on continuous surfaces, one usually solves the differential numerically. Gray [1] presented a numerical scheme of solving the geodesic equation (1) and (2) with $u v$-parametrization using the Mathematica ${ }^{\mathrm{TM}}$ software. Maekawa [2] presented another numerical scheme based on finite difference method. A survey of numerical schemes for solving differential equations and integral equations is available in Cheney [3].

The other class of techniques is used for the polyhedral surfaces. Polthier and Schmies [4] derived the light ray tracing method of shooting the end point of the geodesics along the polyhedral surface. Pham-Trong, Szafran and Biard [5] searched for the discrete geodesics using the subdivision of patches with unfolding, so that the beam propagation can reach the destination point on the other end of the geodesics. Ravi Kumar, Srinivasan, Devaraja Holla, Shastry, and Prakash [6] introduced modified version of Polither and Schmies' work, by incorporating the normal information of the surface. Kimmel and Sethian [7] demonstrated the application of the Fast Matching Method in finding the geodesic paths on a triangularly meshed surface. These methods are not meshless.

In terms of the meshless methods, $\mathrm{Ng}$ and Pong [8] reported the use of distributed collocation method,

\footnotetext{
* This project is financially supported by the Polytechnic University Research Grant, A/C PE04.

+ Contact detail: Phone: +852-2766-6532, Fax:+852-2773-1432, E-mail: roger.ng@polyu.edu.hk

Phone: +852-2766-6935, Fax: +852-2362-9045, E-mail: Glory.Pong@polyu.edu.hk
} 
which is a parallel version of the collocation method. In the current study, another meshless method, radial basis function (RBF), is used in solving the geodesics equations on a family of bicubic tensor product Bezier surface patches under $u v$-parametrization, since interpolation by radial basis functions is a powerful tool in approximation theory.

2) Application of geodesics equation in the apparel industry: In the $3 \mathrm{D}$ garment pattern design, a surface mapping technique, Bijective Pattern Map [9], unfolds the 3D surface patches into 2D enclosed areas using the geodesic information on the 3D mannequin. Manually, the body measurement can be measured by using a tensioned measuring tape. In a virtual world, as a free-form surface, the 3D virtual mannequin can be represented as an atlas of tensor product Bezier or B-Spline or NURBS surface patches. In this case, the body measurement, such as the surface patch shown in Fig. 1, can only be calculated using the geodesic arc length.

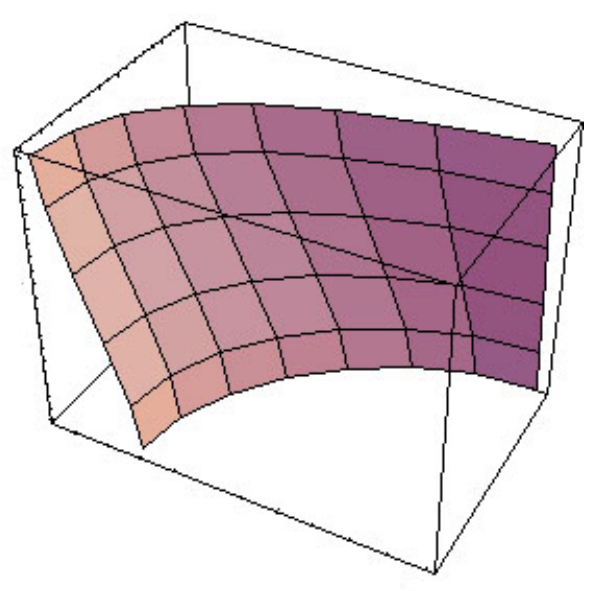

3) Focus of research work: In this article, the calculation of the geodesic will focus on a class of nonself-intersecting surface patches, defined using the bicubic tensor product Bezier form. Self-intersecting surface can be reduced to non-self-intersection surface subpatches. With the scalable properties, it is possible to reduce the problem by considering only the normalized patches, lying within the unit cube. The numerical algorithm is based on the radial basis function method. The choice of the trial functions will be presented. The comparison to the Ritz's method will be given to illustrate the effectiveness of the radial basis function method. Finally, the numerical result will be compared to the data measured from the virtual model of a real mannequin. All the programs are written using Mathematica ${ }^{\mathrm{TM}}$ version 5.0.

\section{NORMALIZATION OF SURFACE PATCHES}

A family of normalized bicubic tensor product Bezier surface patches is defined over the unit cube with the longest side of the patch aligned with the line segment of $[0,1]$ along the $x$-axis. As the bicubic tensor product patch representation is fully scalable, this representation can cover the whole family of bicubic tensor product Bezier surface patches, since any finite bicubic tensor product Bezier surface can be enclosed by a cubic of size equals to the longest straight line distance between any pair of control vertices.

The normalization process can be achieved by these transformations:

Stage 1 (Set origin, and force all points into positive zone):

$$
P_{i}=p_{i}-\operatorname{Min}\left(p_{i}\right)
$$

Stage 2 (Scale to fit the unit cube):

$$
P_{i}^{\mathrm{n}}=P_{i} / \operatorname{Max}\left(P_{i}\right)
$$

where $p_{i}$ are the original data points, $P_{i}$ are the translated points, and $P_{i}^{\mathrm{n}}$ are the normalized points with $\operatorname{Min}($. and $\operatorname{Max}($.$) to be the minimum and maximum function respectively. It should be noted that when the \operatorname{Min}($. is taken over the ordinates individually, the data points are guaranteed to be translated into the positive zone of $\{x, y, z: x \geqq 0 ; y \geqq 0 ; z \geqq 0\}$. However, any of the data points may not be located at the origin. Yet, at least one of the data points can be found on the surface of the unit cube. 


\section{PREPARATION OF COLLOCATION POINTS}

The geodesic from a point $q_{0}$ to another point $q_{1}$ along the bicubic tensor product Bezier surface patch $S(u, v)$ is intended to be written in the form of a parametric curve $\alpha(t)$ with the end points matching the boundary condition of $\alpha(0)=q_{0}$ and $\alpha(1)=q_{1}$. The collocation points are thus defined as the set of $N$ chosen points $\left\{\alpha\left(t_{i}\right)\right.$ where $i=1,2, \ldots, N$, and each $t_{\mathrm{i}}$ lies in the range of $\left.(0,1)\right\}$. In this study, the collocation points are evenly distributed along $(0,1)$. Based on the experience with using collocation method, the collocation points must be chosen in a way to ensure the non-degenerate determinant of the matrix derived by submitting the trial functions and combining the matching requirement at the collocation points.

\section{SELECTION OF TRIAL FUNCTIONS}

The trial functions $g e o_{I}(t)$ and $g e_{2}(t)$ are both selected to be the linear combination of radial basis functions with coefficients $\left\{a_{i}\right\}$ and $\left\{b_{i}\right\}$ respectively, in which we match the conditions available, including the pair of geodesic ODEs, the boundary conditions and the matching at the collocation points. The geo $(t)$ corresponds to the $u$-parameter while the $g^{2} o_{2}(t)$ corresponds to the $v$-parameter. Since the surface is represented in the form of $S(u, v)$, the $G E_{l}(u, v)$ and $G E_{2}(u, v)$ can in fact be written as $G E_{l}(t)=G E_{l}(u(t), v(t))$ and $G E_{2}(t)=G E_{2}(u(t), v(t))$ respectively by substituting:

$$
\begin{aligned}
& \operatorname{geo}_{I}(t)=u(t)=a_{0}+a_{1} \phi\left(t, c_{1}\right)+a_{2} \phi\left(t, c_{2}\right)+a_{3} \phi\left(t, c_{3}\right)+\ldots \\
& \operatorname{geo}_{2}(t)=v(t)=b_{0}+b_{1} \phi\left(t, c_{1}\right)+b_{2} \phi\left(t, c_{2}\right)+b_{3} \phi\left(t, c_{3}\right)+\ldots
\end{aligned}
$$

in which we select the radial basis functions $\phi\left(t, c_{i}\right)[10]$ of the form:

$$
\phi\left(t, c_{i}\right)=\left\{\begin{array}{cc}
\left(1-\frac{d_{i}}{r_{i}}\right)^{5}\left(8+40\left(\frac{d_{i}}{r_{i}}\right)+48\left(\frac{d_{i}}{r_{i}}\right)^{2}+25\left(\frac{d_{i}}{r_{i}}\right)^{3}+5\left(\frac{d_{i}}{r_{i}}\right)^{4}\right), & 0 \leq d_{i} \leq r_{i} \\
0, & d_{i}>r_{i}
\end{array}\right.
$$

where $d_{i}=\left|t-c_{i}\right|$ is the distance between the point $t$ and the center $c_{i}$; and $r_{i}$ is the radius of the support for the radial basis functions centered at the center $c_{i}$. In this study, the radius of the support is chosen to be 2 .

The advantage in using radial basis functions is generally that they provide interpolants irrespective of complexity of the geometry. The compactly supported positive definite radial basis functions $\phi\left(t, c_{i}\right)$ are feasible in the current application. From the experience of approximating the geodesics on the bicubic tensor product Bezier surface patches, radial basis functions must be chosen in a way to ensure the trial functions are at least twice differentiable and with compact support over $[0,1]$.

\section{FORMATION OF MATRIX EQUATION}

In this study, the scope is limited to the geodesics across the diagonal of the surface patch, because such measurements are most commonly used in the Bijective Pattern Map. By substituting the definition of $S(u, v)$, the $g e o_{I}(t)$ and $g o_{2}(t)$ into the pair of geodesics equations, two equations are found, $\mathrm{GE}_{1}(t)$ and $\mathrm{GE}_{2}(t)$. Note that these two equations with the unknown coefficients are very long and are omitted here. By matching the boundary conditions, four more equations are found, (7) to (10).

$$
\begin{aligned}
& u(0)=0=a_{0}+a_{1} \phi\left(0, c_{1}\right)+a_{2} \phi\left(0, c_{2}\right)+a_{3} \phi\left(0, c_{3}\right)+\ldots \\
& v(0)=0=b_{0}+b_{1} \phi\left(0, c_{1}\right)+b_{2} \phi\left(0, c_{2}\right)+b_{3} \phi\left(0, c_{3}\right)+\ldots \\
& u(1)=1=a_{0}+a_{1} \phi\left(1, c_{1}\right)+a_{2} \phi\left(1, c_{2}\right)+a_{3} \phi\left(1, c_{3}\right)+\ldots \\
& v(1)=1=b_{0}+b_{1} \phi\left(1, c_{1}\right)+b_{2} \phi\left(1, c_{2}\right)+b_{3} \phi\left(1, c_{3}\right)+\ldots
\end{aligned}
$$

Finally, requiring a perfect match of the estimation by geo ${ }_{1}(t)$ and geo $2(t)$ at the collocation points $t_{\mathrm{i}}, 2 N$ equations are found, (11) and (12). These equations can be written in the matrix form.

$$
\begin{aligned}
& \mathrm{GE}_{1}\left(t_{i}\right)=0, \mathrm{i}=1, \ldots, \mathrm{N} \\
& \mathrm{GE}_{2}\left(t_{i}\right)=0, \mathrm{i}=1, \ldots, \mathrm{N}
\end{aligned}
$$

After the substitution process is completed, they form a matrix equation. It is important to check and ensure the determinant of this matrix equation to be non-zero, so that solution can be guaranteed. In case, the determinant is zero, the choice of collocation points must be revised.

As an example, from the experience of calculating the geodesics on the bicubic tensor product Bezier 
surface patches when developing the Bijective Pattern Map, two radial basis functions could be sufficient for patches with small Gaussian curvature. In that case, six equations (two from (1) and (2) and four from (5) and (6)) will be derived with two boundary points and one collocation point. When more radial basis functions are used, more collocation points are needed to solve the extra coefficients. If the coefficient matrix of the equation is singular, another set of collocation points falling in between the existing collocation points can be chosen or the number of radial basis functions can be elevated.

\section{DATA PREPARATION}

\subsection{Testing Data}

The testing data is prepared based on the following criteria:

All control vertices are positioned within a unit cube.

The starting endpoint is fixed with the height of 0 ; and the terminating endpoint is with the height of 1 .

The total sample size is 12 points at 6 possible heights $=72$ choices. The control vertices is randomly chosen within a unit cube, but with a predetermined increment, say 0.0225 , while a total 1000 samples are selected. The samples are divided into four groups of data. In this configuration, some of the patches can have local optimal values, however uniqueness of the geodesics is not guaranteed, as conjugate points may exist. Yet, as long as the control points do not intersect each other, the surface patch does not intersect itself. The surface is also ensured to be compact and continuous.

\subsection{Evaluation Data Based on Ritz's Method}

The full set of testing normalized bicubic tensor product Bezier surface patches was run again using another numerical method. Among many choices, the Ritz's method was chosen because it is a popular numerical technique for Calculus of Variation [11]. The basis function for the Ritz's method was chosen to be polynomial in the form of (13) and (14). In this case, the v-parameter becomes a function of u-parameter. The values of geodesic distances were compared to those of the radial basis function method.

$$
\begin{gathered}
u(t)=t \\
v(t)=c_{0}+c_{1} t+c_{2} t^{2}+c_{3} t^{3}+. .+c_{n} t^{n}
\end{gathered}
$$

\subsection{Evaluation Data From Physical Objects}

One more set of data measured from the virtual model of a real mannequin was prepared for comparison. In previous study, the modeling of human mannequin using bicubic tensor product Bezier patches had been demonstrated with reasonable accuracy if the patches were properly defined [12].

The mannequin was digitized by a 3D body scanner and stored in the form of a bicubic tensor product Bezier surface atlas. The lengths of the boundary curves of each patch were ensured to be equal to the physical value. Then, both of the diagonal lengths were measured and used as the reference physical data, and compared to the computer-calculated values.

\section{SOLVING THE MATRIX EQUATION}

The matrix equation derived according to section $\mathrm{V}$ was solved using the built-in equation solver of Mathematica $^{\mathrm{TM}}$ 5.0. However, an adaptive scheme must be used to select different collocation points because it is possible that some pairs of collocation points do share some kind of symmetry, meaning that the rank of the matrix equation may be reduced. In these cases, other collocation points must be used.

It should be noted that the matrix equation is nonlinear with respect to the coefficients $a_{i}$ and $b_{i}$, as the surface patches are in the bicubic tensor product form. Therefore, there can be one or more real solution sets. Each solution set must be testified and compared to determine the global optimal solution set.

During the calculation by Mathematica ${ }^{\mathrm{TM}}$, the response was very slow when three or more radial basis functions were used. So, in this study, the results are based on two radial basis functions centered at $t=1$ and 1.5 with the radius of compact support of 2 .

\section{EVALUATION OF RESULT}

The computation speeds of both methods were measured using the TIMING command of the Mathematica ${ }^{\mathrm{TM}}$. 
Since there are many common codes shared by both methods, the timing of the radial basis function method included the execution of the following lines in pseudo code, see Table I, while the counterpart for the Ritz's Method is listed in Table II.

Table 1 KERNEL OF RADIAL BASIS FUNCTION

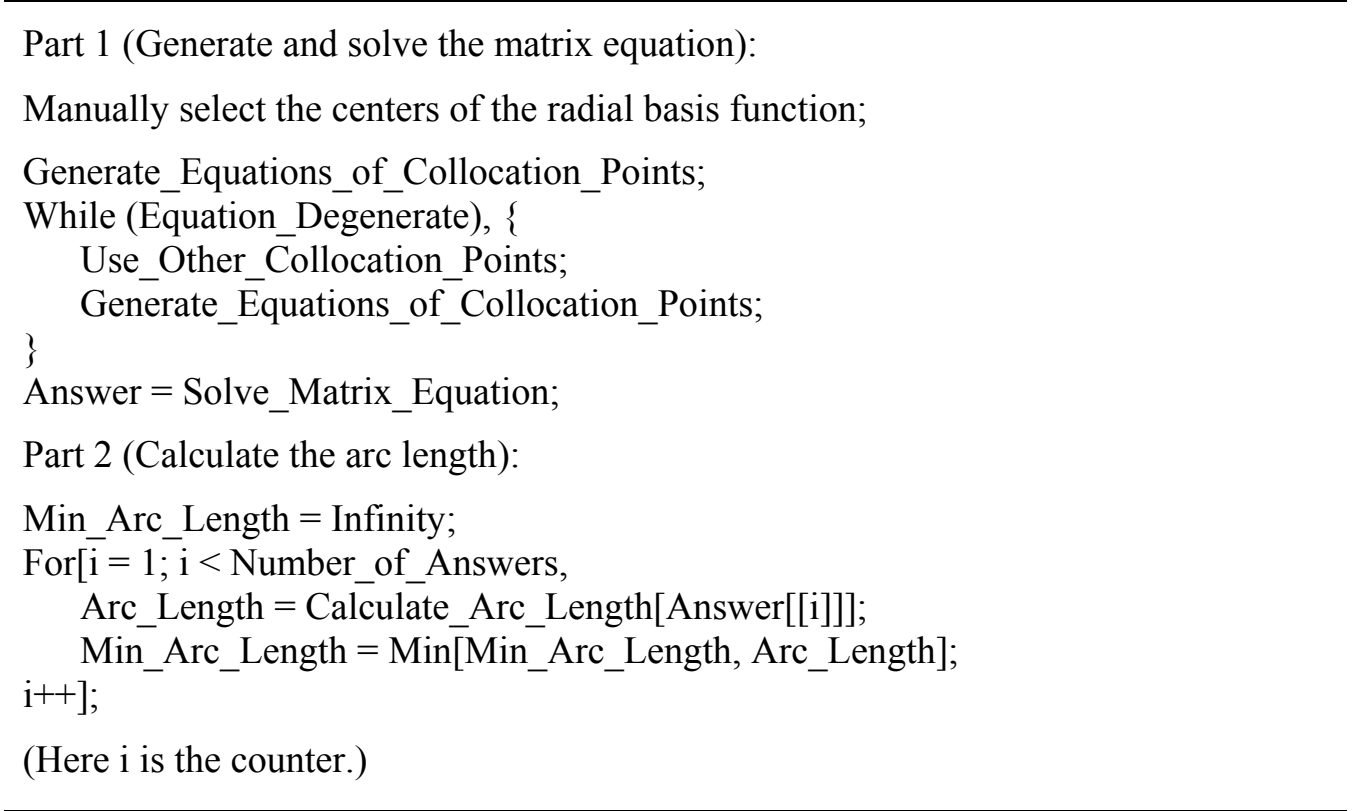

The total computational cost includes the time in generating and solving the matrix equation, and calculating the geodesic distance.

Table 2 KERNEL OF RITZ'S METHOD

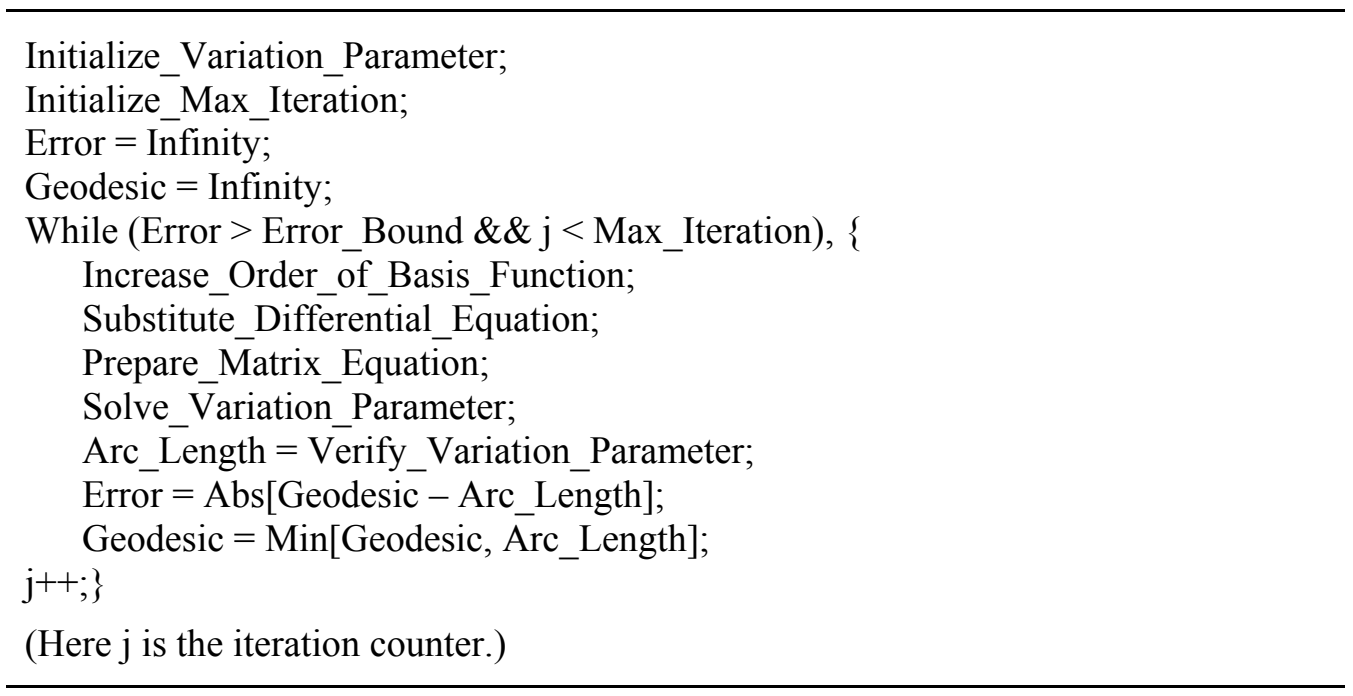

The sample report of the result is listed in Table III. The comparative result between radial basis function method and Ritz's method is summarized in Table IV. The relative Root-Mean-Square error (RMS), (15), is used to indicate the differences in the accuracy of the approximated geodesic distance using radial basis functions, $g C$, and the geodesic distance from Ritz's Method, $g R$, while a relative ratio (16) is used for the improvement of the computation time, $t R$ for Ritz method and $t C$ for radial basis function method. The result is presented in six significant figures in the normalized unit.

$$
\begin{gathered}
\operatorname{Sign}(g C-g R) * \operatorname{Sqrt}\left(\sum\left(((g R-g C) / g R)^{2}\right)\right) \\
t R / t C
\end{gathered}
$$

[Note: Sqrt is the square root function.] 
Table 3 SAMPLE DATA OUTPUT TABLE

\begin{tabular}{cccc}
\hline \multicolumn{2}{c}{ Radial Basis Function } & \multicolumn{2}{c}{ Ritz's Method } \\
\hline Normalized Unit & Second & Normalized Unit & Second \\
1.57495 & 0.141 & 1.56924 & 0.125 \\
1.37739 & 0.141 & 1.37116 & 0.110 \\
1.41368 & 0.125 & 1.40248 & 0.109 \\
1.59956 & 0.140 & 1.59710 & 0.125 \\
\hline
\end{tabular}

Table 4 COMPARISON OF RADIAL BASIS FUNCTION METHOD AND RITZ METHOD

\begin{tabular}{ccc}
\hline Set & Mean Accuracy Loss & Mean Time Improved \\
\hline 1 & $2.89 \%$ & $101 \%$ \\
2 & $3.01 \%$ & $101 \%$ \\
3 & $3.22 \%$ & $98 \%$ \\
4 & $3.17 \%$ & $105 \%$ \\
\hline
\end{tabular}

Finally, a set of sample geodesic distances approximated using the radial basis functions according to the scanned patches was compared to the geodesic distances measured from the virtual model of a real mannequin. The calculated geodesic distances are rounded up to six significant figures. The relative error was measured according to (17), with $g P$ representing physical geodesic. The result is shown in Table V.

$$
\left.\operatorname{Sign}(g C-g P) * \operatorname{Sqrt}((g P-g C) / g P)^{2}\right)
$$

\section{COMMENTS ON USING RADIAL BASIS FUNCTIONS}

Table 5 COMPARISON OF CALCULATION BY RADIAL BASIS FUNCTION METHOD AND PHYSICAL DATA

\begin{tabular}{cccc}
\hline Data & Calculated by RBF Method & Physical Data (cm) & Relative Error (\%) \\
\hline 1 & 13.1038 & 13.0 & -0.8 \\
2 & 6.18354 & 6.25 & 1.06 \\
3 & 10.2788 & 10.25 & -0.28 \\
4 & 8.84254 & 8.75 & 1.06 \\
\hline
\end{tabular}

According to the current pilot study, the advantages of using the radial basis functions in calculating geodesic distances include:

- Efficient method with reasonably accuracy even when small set of radial basis functions to be used.

- Ease of software implementation.

Yet, this method has the following disadvantages: 
- Efficiency drops when more collocation points are used.

- The convergence of the matrix equation solving may take much longer time when more radial basis functions are used.

The typical computation of radial basis function method based on based on two radial basis functions, implemented on a dual Xeon ${ }^{\mathrm{TM}} 1.8 \mathrm{GHz}$ personal computer under Mathematica ${ }^{\mathrm{TM}}$ Version 5.0. This method can be very time consuming as the number of the radial basis functions increases.

\section{CONCLUSION}

In the pilot study, the geodesic equations on the subset of bicubic tensor product Bezier patches have been solved numerically by the interpolation using radial basis functions. The results of computing the geodesics by this radial basis function method are compared to that by the Ritz's method. The agreement between these two methods is acceptable, while the computation efficiency of the radial basis function method is fairly good.

Moreover, the accuracy of approximating geodesics on the scanned patches is found excellent when compared with the measurements on the virtual model of a real mannequin.

The future work is to extend the study to at the full scale, with the theoretical analysis, and weak-form solutions.

\section{References}

[1] A. Gray. Modern Differential Geometry of Curves and Surfaces. CRC Press, 2006.

[2] T. Maekawa. Computation of shortest paths on free-form parametric surfaces. Journal of Mechanical Design. 1996, 118: $499-508$.

[3] W. Cheney. Analysis for Applied Mathematics. New York: Springer-Verlag, 2001.

[4] K. Polthier, M. Schmies. Straightest geodesics on polyhedral surfaces, In: H. C. Hege, H. K. Polthier (Eds.). Mathematical Visualization. Springer-Verlag, 1998.

[5] V. Pham-Trong, N. Szafran, L. Biard. Pseudo-geodesics on three-dimensional surfaces and pseudo-geodesic meshes. Numerical Algorithms. 2001, 26: 305-315.

[6] G.V.V. Ravi Kumar, P. Srinivasan, V. Devaraja Holla, K.G. Shastry, B.G. Prakash. Geodesic curve computations on surfaces. Computer Aided Geometric Design. 2003, 20: 119-133.

[7] R. Kimmel and J.A. Sethian. Computing geodesics paths on manifolds. Applied Mathematics. 95: 8431-8435.

[8] R. NG, G.T.Y. Pong. Measuring Geodesic Body Measurements with Distributed Collocation Method. IMAC 2005. T1-I-45-0374, 2005,

[9] R. NG, C. K. CHAN, R. AU, T.Y. PONG. Analytical solution to the Bijective Mapping Problem in pattern design. Journal of China Textile University. (English Edition), 1996, 13: 94-98.

[10]Z. Wu. Compactly supported positive definite radial functions. Advances in Computational Mathematics. 1995, 4: 283-292.

[11]I. M. Gelfand, S. V. Fomin. Calculus of Variation. New Jersey: Prentice Hall, 1963.

[12] R. NG, C. K. CHAN, R. AU, T.Y. PONG. Automatic generation of human model from linear measurements Algebraic Mannequin. Ergon-Axia '98. 1998, 267-270.

\section{TRADEMARK}

Mathematica $^{\mathrm{TM}}$ is a trademark of Wolfram Research Inc.

$\mathrm{Xeon}^{\mathrm{TM}}$ is a trademark of Intel Corporation. 
